\title{
Diacronie
}

Studi di Storia Contemporanea

$N^{\circ} 25,1 \mid 2016$

"Se creare è definire"

\section{Gabriele Balbi, Paolo Magaudda, Storia dei media digitali. Rivoluzioni e continuità}

\section{Luca Zuccolo}

\section{OpenEdition}

Journals

\section{Edizione digitale}

URL: http://journals.openedition.org/diacronie/3900

DOI: 10.4000/diacronie.3900

ISSN: 2038-0925

Editore

Association culturelle Diacronie

Notizia bibliografica digitale

Luca Zuccolo, "Gabriele Balbi, Paolo Magaudda, Storia dei media digitali. Rivoluzioni e continuità »,

Diacronie [Online], № 25, 1 | 2016, documento 15, online dal 29 mars 2016, consultato il 10 décembre 2020. URL : http://journals.openedition.org/diacronie/3900 ; DOI : https://doi.org/10.4000/diacronie. 3900 


\section{RECENSIONE:}

\section{Gabriele BALBI, Paolo MAgAUDDA, Storia dei media digitali. Rivoluzioni e continuità, Roma-Bari, Laterza, 2014, XXI + 181 pp.}

a cura di Luca ZUCCOLO *

Al giorno d'oggi ci confrontiamo quotidianamente con i media digitali, li usiamo - a volte ne abusiamo - li critichiamo e li confrontiamo con i media "tradizionali", ma, spesso, poco conosciamo della loro storia e dei loro aspetti culturali, ovvero «del rapporto tra le tecnologie mediali e le strutture culturali e simboliche più profonde di una società »'.

La storia "socio-culturale" dei media digitali, la digitalizzazione come rivoluzione e al tempo stesso continuità in un processo storico che va ben al di là della semplice modificazione del nostro modo di accedere alle informazioni o di comunicare è al centro di questo volume, che unisce le competenze di Gabriele Balbi, professore assistente in Media Studies presso l'Università della Svizzera italiana di Lugano, e Paolo Magaudda, sociologo presso l'Università di Padova. Il saggio qui presentato si inserisce, infatti, in un ampio filone di ricerche storiche e sociologiche sui media digitali e sulle loro interazioni con la società presente a cui i due autori hanno contribuito con i loro precedenti volumi, tra cui ricordiamo il saggio di Balbi sulle origini del telefono in Italia e quello di Magaudda sul consumo delle tecnologie musicali².

$\mathrm{Si}$ è introdotto così il tema della storia socio-culturale, ma il volume di Balbi e Magaudda oltre ad analizzare tre dei principali media digitali - il personal computer, il telefono portatile e internet - sviluppa un altro interessante filone di ricerca ed analisi: il concetto di digitale e la digitalizzazione dei media analogici. Questi due temi, spesso

1 BALBI, Gabriele, MAGAUDDA, Paolo, Introduzione, in IID., Storia dei media digitali, Rivoluzioni e continuità, Roma-Bari, Laterza, 2014, p. XVII.

2 BALBI, Gabriele, Le origini del telefono in Italia, Milano, Mondadori, 2011; MAGAUDDA, Paolo, Oggetti da ascoltare, HiFi, iPod e consumo delle tecnologie musicali, Bologna, Il Mulino, 2012. 
appiattiti sull'oggi e sui loro aspetti rivoluzionari vengono rivisitati in relazione alla storia dei tre media testé citati sottolineando come gli aspetti rivoluzionari si intersecano con una continuità storica di Longue durée che, analizzata ottimamente dai due autori in tutti i suoi aspetti, testimonia una profondità storica del fenomeno digitale strettamente connessa agli aspetti socio-culturali delle "comunità" in cui i media digitali sono nati e si sono sviluppati.

Il volume, infatti, è ricco di esempi di questo reciproco rapporto, tra cui possiamo segnalare il mondo e la cultura degli hacker sviluppatasi in California negli anni Sessanta e Settanta che ha consentito lo sviluppo del personal computer e della rete internet come li conosciamo oggi. La contro-cultura emersa nei circoli degli hobbisti della Baia di San Francisco e in particolare lo Homebrew Computer Club, unita alle spinte dei movimenti per i diritti civili e alla contro-cultura giovanile degli anni Settanta, produssero la nuova visione del personal computing che, grazie a personaggi come Steve Jobs e Steve Wozniak, permisero l'accesso alle nuove tecnologie da parte delle masse 3 .

Il digitale come ossessione dei tempi moderni, quindi, come viene spiegato nel primo capitolo, si sviluppa in un quadro di lunga durata. Periodi "rivoluzionari" che si collegano strettamente sia alle società in cui il digitale si è e si sta sviluppando, sia agli aspetti politici di queste stesse realtà, ne condizionano o stimolano la relazione con le nuove tecnologie.

Su queste basi, il volume propone nei tre capitoli centrali - secondo, terzo e quarto - lo studio della storia evolutiva e delle strette relazioni con gli aspetti sociali e politici di tre media peculiari: il computer, internet e il telefono mobile.

Come visto poco sopra, la storia del computer è ricca di spunti tratti dalla società in cui i personal devices si sono sviluppati passando da mega strutture militari governative a macchine personali e personalizzate. Il capitolo secondo analizza questa relazione andando ad approfondire come le possibilità tecniche sviluppatesi a partire dai mainframe si siano rapportate con gli utenti, con le loro esigenze e come questa relazione abbia modificato il computer fino a renderlo dapprima un oggetto di consumo e poi un device portatile, facendo la fortuna sia della Apple di Steve Jobs, sia della Microsoft di Bill Gates. Due attori chiave dell'evoluzione del PC dal punto di vista dell'hardware e del software e due figure centrali negli ultimi trent'anni anche nell'interconnessione tra PC e gli altri media digitali a iniziare da internet, tema del capitolo successivo.

3 BALBI, Gabriele, MAGAUDDA, Paolo, op. cit., pp. 29-30. 
Il terzo capitolo, infatti, si concentra sullo sviluppo della rete internet da Arpanet fino alle sue versioni odierne. Il capitolo propone un analisi del medium attraverso le sei "ere", della sua evoluzione «ognuna delle quali è caratterizzata da altrettante influenze socio-culturali»4.

Queste ere, che si sviluppano dalla fine degli anni Cinquanta fino ad oggi, non devono essere interpretate soltanto in termini diacronici e consecutivi, ma come differenti dimensioni che si sono stratificate e che continuano a convivere le une con le altre. Esse hanno avuto un ruolo determinante nello sviluppo politico, economico e tecnologico di internet, contribuendo a co-costruire alcuni dei significati ancora oggi alla base di ciò che definiamo come internet5.

La rete come la conosciamo noi, tuttavia, non è l'unica e i due autori propongono un interessante confronto tra due reti alternative: quella francese del Minitel e quella cinese, basata su uno stretto controllo. Questi due esempi giustificano l'ampiezza e la varietà della storia di internet analizzando aspetti centrifughi e non convenzionali, chiarendo come la rete internet che noi usiamo e conosciamo sia solo una delle possibili evoluzioni e varianti possibili.

Il quarto capitolo sposta l'attenzione su un altro media fondamentale per la nostra società contemporanea e sempre più invasivo nei numeri e nelle sue funzioni tanto da assorbire molte se non tutte le peculiarità dei due strumenti precedentemente descritti in questo libro: il telefono mobile.

Gli autori non solo analizzano lo sviluppo del telefono portatile e dei suoi differenti sviluppi in America ed in Europa, ma collegano questa evoluzione tecnica a peculiari dinamiche socio-politiche che fecero rinunciare la AT\&T, azienda monopolista delle comunicazioni negli USA, a puntare sul nuovo dispositivo, così come permisero una sua ampia diffusione in Europa grazie ad un vero e proprio "miracolo burocratico" quando nel 1992 molti paesi si adeguarono alla rete gsm.

Infine, il quinto capitolo, descrive la digitalizzazione dei media analogici: musica, stampa, cinema, fotografia, televisione e radio; ovvero l'interazione tra gli sviluppi tecnologici descritti nei capitoli precedenti e questi sei settori della comunicazione e della cultura ${ }^{6}$.

La digitalizzazione dei media analogici è descritta secondo due logiche di analisi. La prima riconosce la digitalizzazione come un semplice processo tecnico e materiale che

${ }^{4}$ Ibidem, p. 46.

5 Ibidem.

${ }^{6}$ Ibidem, p. 100. 
trasforma i media classici in media digitali. La seconda, invece, analizza il più complesso processo di "rimediazione" e riconfigurazione dei media analogici sia nei contenuti che nelle loro pratiche culturalì. Un esempio su tutti è senza dubbio la nuova stagione dei vinili a 33 giri che, pur restando un fenomeno di nicchia, stanno dando nuova linfa a questo primigenio media musicale.

Il volume si conclude con un ultimo capitolo, I "miti fondativi" del digitale tra rivoluzione e continuità, in cui i due autori - riprendendo quanto sostenuto lungo tutto il saggio - propongono una loro visione dei miti sul digitale sulla rivoluzione digitale, riconsiderandola alla luce di processi sociali e culturali molto più ampi.

I media hanno infatti prodotto conseguenze profonde e possono portare in futuro, intrecciandosi con nuove istanze tecniche, culturali e sociali, a effettivi salti nell'esperienza, nelle capacità o forse addirittura nell'antropologia umana. Ma, d'altro canto, la logica della "cesura netta", del cambio improvviso e repentino di paradigma, è altrettanto fuorviante perché la comprensione dei cambiamenti socioculturali richiede di mettere nella prospettiva di una longue durée gli intrecci tecnici, culturali e sociali nel campo dei media ${ }^{8}$.

Proponendo quindi il concetto di "rivoluzione conservativa" Balbi e Magaudda concludono il loro lavoro offrendo in appendice una ricca bibliografia e una serie molto utile di dati quantitativi sui media digitali che arricchiscono un volume adatto al grande pubblico e fondamentale per gli studiosi di nuove tecnologie.

Nel mare magnum degli studi sui nuovi media il volume in oggetto si propone come un utile strumento, di facile lettura, e dotato di quella profondità analitica che consente al lettore di districarsi tra le molteplici teorie e ipotesi legate al mondo digitale. Merito dei due autori, infatti, è quello di fare chiarezza su alcuni processi storici ed evolutivi di questi media proponendo una visione innovativa degli stessi, non più appiattita sui soli aspetti rivoluzionari e di cesura. Puntando, invece, sugli aspetti di continuità e sulle molteplici articolazioni e relazioni culturali di questi media i due autori ci propongono un'analisi storico-sociologica di grande spessore.

\footnotetext{
7 Ibidem.

8 Ibidem, p. 151. 


\section{* L'autore}

Luca Zuccolo, dottore (PhD) in Storia Contemporanea del SUM di Napoli ha sviluppato una ricerca sulle dinamiche di formazione dell'identità ottomana e di un discorso di tipo patriottico durante il regno di Abdülhamid II (1880-1885) attraverso lo studio di fonti a stampa redatte in lingua francese. Già dottore magistrale in Storia d'Europa (Bologna 2008), si è occupato dello sviluppo della modernità durante l'ultimo secolo dell'Impero Ottomano, del confronto/scontro tra modernità e tradizione in un contesto cosmopolita e allo sviluppo dei movimenti sociali che hanno preparato l'avvento della società turca contemporanea.

URL: <www.studistorici.com/2010/12/o1/luca-zuccolo >

\section{Per citare questo articolo:}

ZUCCOLO, Luca, «Recensione: Gabriele BALBI, Paolo MAGAUDDA, Storia dei media digitali. Rivoluzioni e continuità, Roma-Bari, Laterza, 2014, XXI + 181 pp.», Diacronie. Studi di Storia Contemporanea: "Se creare è definire", 29/03/2016,

URL:< http://www.studistorici.com/2016/03/29/zuccolo_numero_25/ >

\section{Diacronie Studi di Storia Contemporanea $\beta$ www.diacronie.it}

Risorsa digitale indipendente a carattere storiografico. Uscita trimestrale. redazione.diacronie@hotmail.it

Comitato di redazione: Jacopo Bassi - Luca Bufarale - Elisa Grandi - Antonio César Moreno Cantano - Deborah Paci - Fausto Pietrancosta - Alessandro Salvador - Matteo Tomasoni - Luca Zuccolo

Diritti: gli articoli di Diacronie. Studi di Storia Contemporanea sono pubblicati sotto licenza Creative Commons 3.0. Possono essere riprodotti e modificati a patto di indicare eventuali modifiche dei contenuti, di riconoscere la paternità dell'opera e di condividerla allo stesso modo. La citazione di estratti è comunque sempre autorizzata, nei limiti previsti dalla legge. 